experience in the use of this drug for this condition in almost 400 patients has been reported by Hammerstein et $a l^{1}$ and they noted significant improvement in hirsuties in the majority of cases. They recommend a "reverse sequential" regimen using a dose of $100 \mathrm{mg}$ of cyproterone acetate on days $5-14$ of the cycle, combined with $50 \mu \mathrm{g}$ of ethinyl oestradiol on days 5-25. The latter drug is added for cycle control and certain contraception.

Using this regimen we have demonstrated a marked reduction in the rate of growth as well as the diameter of the individual hairs. ${ }^{2}$ Beneficial results with this drug have also been reported by Barnes et al. ${ }^{3}$

The drug is primarily a competitive inhibitor of androgens at the receptor level, ${ }^{4}$ but it also inhibits steroid synthesis. ${ }^{5}$ Because of this effect caution is necessary in its use, and adrenal insufficiency has been reported in children being treated for precocious puberty. ${ }^{6}$ However, the study by Hammerstein et al found no evidence of adrenal impairment.

Your comments on women's attitude to excessive body hair are more optimistic than our current experience indicates and perhaps rather flippant. We have found that, although the condition is not serious medically, these women are extremely anxious and agitated by their condition, sometimes seeking psychiatric assistance. Certainly the necessity for frequent shaving is very distressing to them. Their mood dramatically changes, however, when they can see the benefits from the treatment.

ADRIAN K THOMAS

Department of Obstetrics and Gynaecology,
University of Sheffield

'Hammerstein, J, et al, Fournal of Steroid Biochemistry, 'Hammerstein,

'Thomas, A K, Ebling, F J, and Cooke, I D, British Fournal of Obstetrics and Gynaecology, in press.

Barnes, E W, et al, Clinical Endocrinology, 1975, 4, 65 Neumann, F, and Graf, K-J, Journal of International Medical Research, 1975, 3, supplement (4) 1 .

Panesar, N S, and Stitch, S R, fournal of Endocrinology, 1976, 69, 3, 14P.

crinology, 1976, 69, 3, $13 \mathrm{P}$.

\section{Art of general practice}

SIR,-May I welcome your new series "Clinics in General Practice." The consultant's comments were excellent and succinctly made. The trainer, with reference to diarrhoea and pyrexia, has missed the teaching point of the case. The art of general practice is to remember that "common things are common," but the wise GP keeps the small print diagnosis up his sleeve. In small children one of the more common causes of diarrhoea and pyrexia is upper respiratory infection. One of the rarer (besides intestinal infections of salmonella nature) is appendicitis.

DeREK A COFFMAN

Wembley,

Middlesex

\section{Reliable detection of ruptured membranes}

SIR,-Many antenatal patients are hospitalised because of doubt as to whether there has been a spontaneous leak of liquor. Furthermore, their management as regards early delivery may also depend on the definitive knowledge as to whether the membranes are intact. Tests to differentiate between the alkalinity of liquor and the acidity of vaginal fluid and urine were described in the 1930s. ${ }^{12}$ However, our inquiries suggest that few obstetricians use such tests and many were unaware of them. We have therefore carried out a study of the efficacy of sterile nitrazine yellow-coated swabs mounted on sticks (Medical Wire and Equipment Co (Bath) Ltd). These swabs indicate a change to an alkaline $\mathrm{pH}$ by turning blue when even a small amount of liquor is present at the external cervical os.

A correct positive result was obtained in 75 patients whose membranes had been ruptured artificially one to four hours earlier and a correct negative result was obtained in 74 patients who were swabbed at the antenatal clinic during the third trimester. In one further antenatal patient a negative result was obtained during premature labour, but the membranes were found to be clinically ruptured 48 hours later. Other swabs were taken from patients with blood (13) and meconium (8) stained liquor, and a positive result was still obtained. Swabs from antenatal and gynaecological patients with proved trichomonal or monilial vaginitis did not give clinically false results. Lastly, vaginal swabs were taken in the presence of Hibitane cream, Hibitane solution, and $\mathrm{KY}$ jelly, and again clinically false results were not obtained.

We believe that nitrazine yellow swabs provide a very cheap and effective way of differentiating a leak of liquor from urine or vaginal discharge. We recommend a cervical swab taken by a sterile technique rather than a low vaginal swab, as it is possible that contamination of the lower vagina with urine (which occasionally is alkaline) may give a false positive result, and similarly a low vaginal swab may miss small amounts of liquor at the cervix.

E SKLOVSKY Clinical assistant

\section{A H MacLennan} Lecturer

Nuffield Department of Obstetrics and Gynaecology, John Radic

${ }^{1}$ Berlind, M M, American Fournal of Obstetrics and Gynecology, 1932, 24, 198.
King, A G, American Journal of Obstetrics and
Gynecology, 1935, 30, 860.

\section{Curry kidney}

SIR,-I was most interested to read your leading article on the curry kidney (10 July, p 69). You observed on the basis of the two small studies that in Fiji the Indians are "virtually the only sufferers from stones." Further, you suggested that the similarity between the ingredients in Worcestershire sauce and curry may be the clue to the pathogenesis of renal stones. Singapore is a mixed ethnic community with a significant proportion of curry-consuming Indians. A recent study of 254 hospital patients of the different ethnic groups resident in Singapore, ${ }^{1}$ including Indians from different parts of India, failed to demonstrate any statistically significant increase in the incidence of renal calculi among Indians.

There were 49 Indians, 177 Chinese, 24 Malays, and 4 other patients in the study. Indians accounted for $19.3 \%$ of the patients seen while they constituted $7 \cdot 0 \%$ of the population of Singapore. The apparent increase is explained by the fact that the hospital utilisation figures showed that Indians constitute $14.3 \%$ of patients seen. Furthermore, the age and sex distribution of the Indian population in Singapore is peculiar. There is a preponderance of Indian males in the 20 to 59 age group in the population. This is the population at increased risk of renal stone disease. ${ }^{2}$

Multiethnic communities are interesting sources of epidemiological information, but enthusiasm in interpretation of small numbers studied has to be tempered by a statistical analysis which takes in important variables. While curries and Worcestershire sauce may continue to spice the aetiology of renal stone disease, the meat of the matter still escapes our notice.

Singapore General Hospital

J A TAMBYAH

'Tambyah, J A, et al, Singapore Medical fournal, 1972,
6, 269.
2avan, J N, et al, Medical fournal of Australia, 1971,
2, 1049.

\section{Hazard of glutethimide}

SIR,-A patient with osteomalacia was referred by $\operatorname{Dr} G R$ Clarke when working in this department for further investigation in the department of metabolic diseases. She was detected as having hepatic enzyme induction leading to rapid vitamin $\mathrm{D}$ destruction caused by prolonged glutethimide administration. ${ }^{1}$ This hazard had not been previously reported but is an additional contraindication to prolonged administration of glutethimide and possibly barbiturates not mentioned in your leading article (12 June, $\mathrm{p}$ 1424).

J A Mathews D A H YATES Department of Rheumatology,
St Thomas's Hospital,

' Greenwood, R H, Prunty, F T G, and Silver, J, British Medical fournal, 1973, 195, 643 .

\section{Hormonal pregnancy tests and} congenital malformations

SIR,-According to a reliable medical source some general practitioners are still using hormonal pregnancy tests despite the warning notice issued last year by the Committee on Safety of Medicines. ${ }^{1}$ In view of the possible fetal damage which might be induced with this method of pregnancy diagnosis ${ }^{2-4}$ the information requires thorough checking-even if it is based on hearsay evidence. This practice may have arisen from certain confusing factors.

The warning notice referred to 12 different preparations which were used for pregnancy testing, of which only Primodos and Norlestrin are available at present. The former product (manufactured by Scherings Chemicals, Ltd) has been recognised primarily for pregnancy testing, and proved to be very popular, ${ }^{2}$ whereas the latter one (a Parke-Davis product) had the reputation of being an oral contraceptive and was less frequently used for diagnostic purposes. These products are now recommended for the symptomatic treatment of secondary amenorrhoea, and the manufacturers' data sheets have been revised accordingly. ${ }^{5}$

The fact that these two products remained on the market while the others were withdrawn may formulate false confidence. The warning remarks on the adverse effect in pregnancy, 